\title{
A STUDY OF 3- AND 4-BODY HADRONIC FINAL STATES USING INITIAL STATE RADIATION WITH BABAR
}

\author{
E. P. SOLODOV \\ Representing the BABAR Collaboration \\ Budker Institute of Nuclear Physics, 11 Lavrentieva, Novosibirsk 630090, Russia \\ E-mail: E.P.Solodov@inp.nsk.su
}

\begin{abstract}
A study of several 3- and 4-body hadronic final states $\left(\pi^{+} \pi^{-} \pi^{0}, \pi^{+} \pi^{-} \pi^{+} \pi^{-}, K^{+} K^{-} \pi^{+} \pi^{-}\right.$and $\left.K^{+} K^{-} K^{+} K^{-}\right)$accompanied by hard photon is presented. These states are produced from $e^{+} e^{-}$ collisions at the c.m. energy near $\Upsilon(4 S)$ resonance using $90 \mathrm{fb}^{-1}$ data sample collected with the BABAR detector at the PEP-II colider. The invariant mass of the hadronic final state determines the virtual photon energy, so that data can be compared with direct $e^{+} e^{-}$cross sections. The cross sections for the above final states have been obtained from the threshold to $4.5 \mathrm{GeV}$ with about $5 \%$ systematic errors. The accuracy of the results are comparable with the best direct $e^{+} e^{-}$results overall and with much better precision in 1.4-2.5 GeV region where very few data are available. In addition to light meson spectroscopy these data can be used to improve the determination of $\mathrm{R}$ - the ratio of $e^{+} e^{-} \rightarrow$ hadrons cross section to $e^{+} e^{-} \rightarrow \mu^{+} \mu^{-}$- and thereby to impact the understanding of recent $(g-2)_{\mu}$ measurement. The ISR technique also gives access to $J / \psi$ production. Measurements of branching ratios into 3- and 4-body final states given above are made to a level of precision that is tipically better than that obtained in the combined earlier measurements.
\end{abstract}

\section{Introduction}

The possibility of using the initial state radiation (ISR) of hard photons at B-factories to study hadronic final state production at lower $e^{+} e^{-}$c.m. energies has been discussed previously ${ }^{1,2,3}$. The interest of this kind of study has been increasing because of discrepancy between the measured muon g-2 value and the one predicted by the Standard Model ${ }^{4}$, where the hadronic contribution to the prediction is taken from $e^{+} e^{-}$ experiments at low energies. The study of ISR events at B-factories can provide independent measurements of hadronic cross sections as well as contribute to low mass resonance spectroscopy.

The ISR cross section for a particular final state $f$ depends on $e^{+} e^{-}$cross section $\sigma_{f}(s)$ and is obtained from:

$$
\frac{d \sigma(s, x)}{d x}=W(s, x) \cdot \sigma_{f}(s(1-x)),
$$

where $\quad x=\frac{2 E_{\gamma}}{\sqrt{s}} ; E_{\gamma}$ is the energy of the ISR photon in the nominal c.m. frame, and $\sqrt{s}$ is the nominal c.m. energy. The function $\mathrm{W}(\mathrm{s}, \mathrm{x})$ describes the energy spectrum of the virtual photons and can be calculated with better than $1 \%$ accuracy ${ }^{1,2,3}$. ISR photons are produced at all angles relative to the collision axis. The BABAR acceptance for such photons is around $10-15 \%{ }^{3}$.

An advantage deriving from the use of ISR is that the entire range of effective collision energy is scanned in one experiment. This avoids the relative normalization uncertainties which can arise when data from different experiments are combined. A disadvantage is that the invariant mass resolution limits the width of the narrowest structure which can be measured via ISR production.

The ISR method gives an access to $J / \psi$ decays. The cross section for the final state $f$

$\sigma_{J / \psi}^{f}(s)=\frac{12 \pi^{2} \Gamma_{e e} B_{f}}{m_{J / \psi} \cdot s} \cdot W(s, x) ; x=1-\frac{m_{J / \psi}^{2}}{s}$,

is proportional to the product $\Gamma_{e e} \cdot B_{f}$ or $\Gamma \cdot B_{e e} \cdot B_{f}$ where $\Gamma$ and $B_{e e}, B_{f}$ are the total width and branching fractions of $J / \psi$ to $e^{+} e^{-}$and $f$. The invariant mass of the final particles determines the position of $J / \psi$ peak and a detector mass resolution 
$\sim 8 \mathrm{MeV}$ can be achieved by using a kinematic fit. Preliminary studies of some particular ISR processes have been performed (see Ref. ${ }^{5,6,7}$ ) showing good BABAR detector ${ }^{8}$ efficiency and particle identification capability for these type of events. Signal and background ISR processes are simulated using Monte Carlo (MC) event generators based on the computer code described in Ref. ${ }^{9,10,11,12}$. Also simulated were generic background from quark-antiquark and $\tau \bar{\tau}$ processes using JETSET ${ }^{18}$ and KORALB ${ }^{19}$ packages.

\section{$2 \pi^{+} \pi^{-} \pi^{0}$ final state}

The initial selection of $e^{+} e^{-} \rightarrow \pi^{+} \pi^{-} \pi^{0} \gamma$ candidates requires that all the final particles are detected inside a fiducial volume. One of the photons is required to have an energy in the c.m. frame above $3 \mathrm{GeV}$. Two of the tracks must originate from the interaction point, have a transverse momentum above $100 \mathrm{MeV} / c$ with no kaon-ID and be in the good region of detector acceptance. The photon with greatest c.m. energy is assumed to be the ISR photon. The remaining photons are paired to form candidate $\pi^{0} \mathrm{~s}$. A kinematic fit is applied to the selected event, imposing energy and momentum conservation, and constraining the candidate $\pi^{0}$ invariant mass. Analysis details can be found in Ref. ${ }^{7}$. The $3 \pi$ invariant mass distribution of the events after background subtraction was fit with a sum of excitation curves describing $\omega(782), \phi(1020), \omega(1420)$ and $\omega(1650)$ resonances. Relative phases for $\omega(1420)$ and $\omega(1650)$ are fixed at 0 and $\pi$. The resulting parameters obtained from the fit (Fig. 1, $\chi^{2} /$ dof $\left.=146 / 148\right)$ are the following:

$\mathcal{B}_{\omega e e} \mathcal{B}_{\omega 3 \pi}=(6.70 \pm 0.06 \pm 0.27) \times 10^{-5}$, $\mathcal{B}_{\phi e e} \mathcal{B}_{\phi 3 \pi}=(4.30 \pm 0.08 \pm 0.21) \times 10^{-5}$, $\mathcal{B}_{\omega^{\prime} e e} \mathcal{B}_{\omega^{\prime} 3 \pi}=(0.82 \pm 0.05 \pm 0.06) \times 10^{-6}$, $M_{\omega^{\prime}}=(1350 \pm 20 \pm 20) \mathrm{MeV} / c^{2}$, $\Gamma_{\omega^{\prime}}=(450 \pm 70 \pm 70) \mathrm{MeV} / c^{2}$, $\mathcal{B}_{\omega^{\prime \prime} e e} \mathcal{B}_{\omega^{\prime \prime} 3 \pi}=(1.3 \pm 0.1 \pm 0.1) \times 10^{-6}$, $M_{\omega^{\prime \prime}}=(1660 \pm 10 \pm 2) \mathrm{MeV} / c^{2}$,
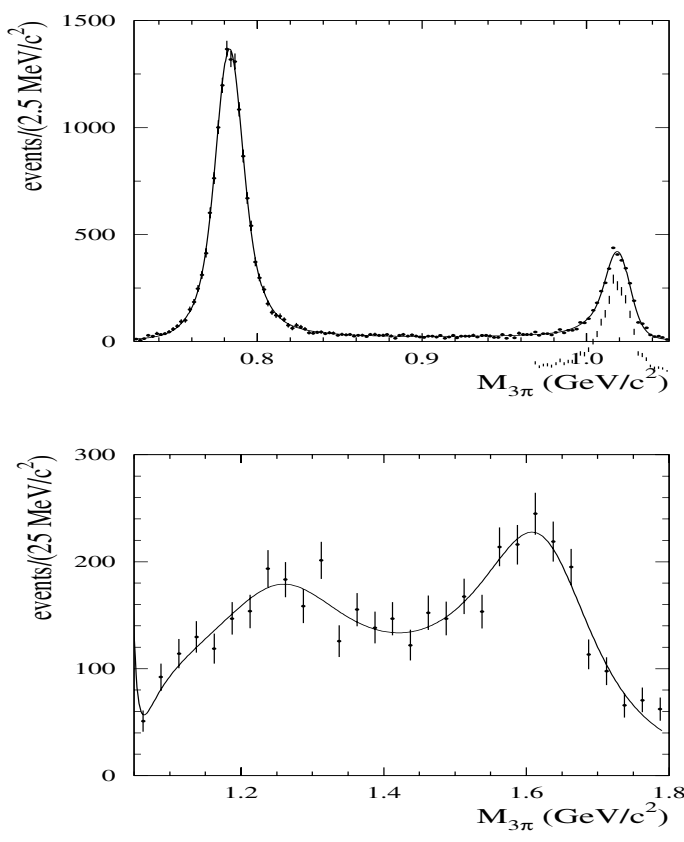

Figure 1. The background-subtracted $3 \pi$ mass spectrum for masses between 0.70 and $1.05 \mathrm{GeV} / c^{2}$ (top) and for masses from 1.05 to $1.80 \mathrm{GeV} / c^{2}$ (bottom). The curves are the result of the fit.

$\Gamma_{\omega^{\prime \prime}}=(230 \pm 30 \pm 20) \mathrm{MeV} / c^{2}$.

The fitted masses and widths of the $\omega^{\prime}$ and $\omega^{\prime \prime}$ mesons are model dependent but nevertheless can be compared with the estimates of these parameters by the PDG ${ }^{14}: M_{\omega^{\prime}}=1400-$ $1450 \mathrm{MeV} / c^{2}, \quad \Gamma_{\omega^{\prime}}=180-250 \mathrm{MeV} / c^{2}$, $M_{\omega^{\prime \prime}}=1670 \pm 30 \mathrm{MeV} / c^{2}, \Gamma_{\omega^{\prime \prime}}=315 \pm$ $35 \mathrm{MeV} / c^{2}$. The PDG data are based on small data samples for $e^{+} e^{-} \rightarrow \omega^{\prime}, \omega^{\prime \prime} \rightarrow$ $3 \pi, \omega \pi \pi^{16,15,20}, p \bar{p} \rightarrow \omega^{\prime} \pi^{0} \rightarrow \omega \pi^{0} \pi^{0} \pi^{0}{ }^{21}$, and $\pi^{-} p \rightarrow \omega^{\prime \prime} n \rightarrow \omega \eta n{ }^{22}$ reactions. We present a new measurement of the $\omega^{\prime}$ and $\omega^{\prime \prime}$ parameters based on a significantly larger data sample for the $e^{+} e^{-} \rightarrow \omega^{\prime}, \omega^{\prime \prime} \rightarrow 3 \pi$ reaction. From the measured values of $\mathcal{B}(V \rightarrow$ $\left.e^{+} e^{-}\right) \mathcal{B}(V \rightarrow 3 \pi)$, the electronic widths of $\omega^{\prime}$ and $\omega^{\prime \prime}$ can be estimated. Assuming that $\mathcal{B}\left(\omega^{\prime} \rightarrow 3 \pi\right) \approx 1$ and $\mathcal{B}\left(\omega^{\prime \prime} \rightarrow 3 \pi\right) \approx 0.5$ we derive that $\Gamma\left(\omega^{\prime} \rightarrow e^{+} e^{-}\right) \approx 370 \mathrm{eV}$ and $\Gamma\left(\omega^{\prime \prime} \rightarrow e^{+} e^{-}\right) \approx 570 \mathrm{eV}$. The large values of these widths, comparable with $\Gamma(\omega \rightarrow$ $\left.e^{+} e^{-}\right) \approx 600 \mathrm{eV}$, are in disagreement with ex- 


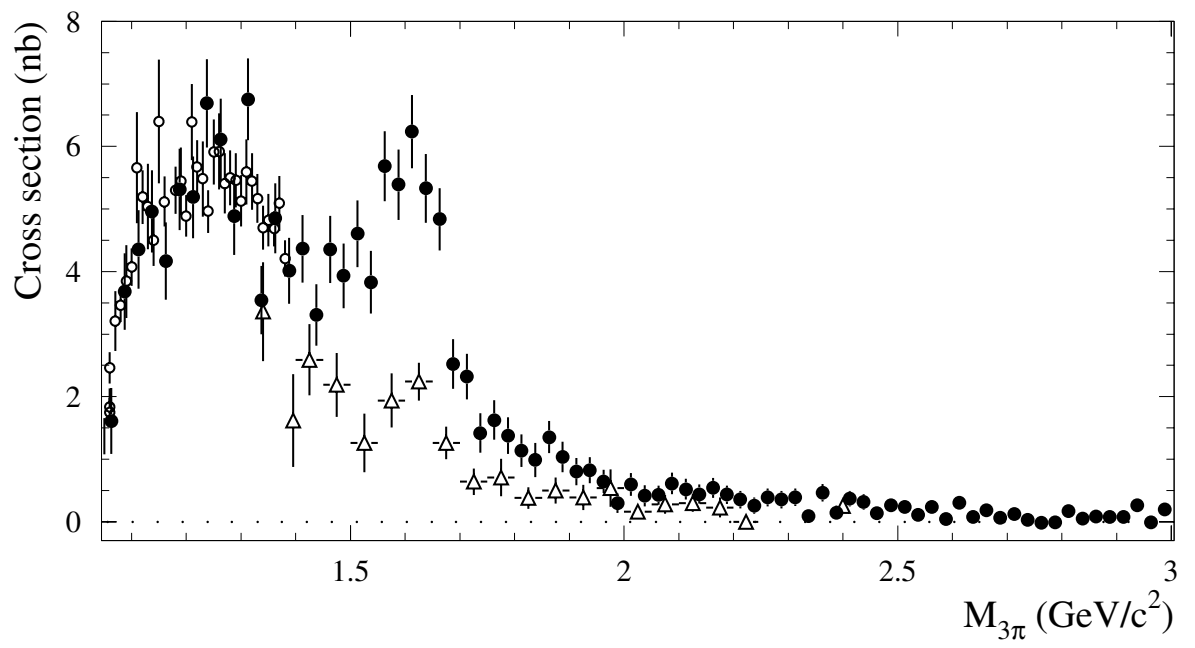

Figure 2. The $e^{+} e^{-} \rightarrow \pi^{+} \pi^{-} \pi^{0}$ cross section measured in this work (filled circles), by SND (open circles), and DM2 (open triangles).

pectations of the quark model, which predicts at least one order of magnitude lower values for the electronic widths for the excited meson states (see, for example, Ref. ${ }^{23}$ ).

The $e^{+} e^{-} \rightarrow \pi^{+} \pi^{-} \pi^{0}$ cross section in the 1.05-3.0 GeV/ $c^{2}$ region is presented in Fig. 2. It is in agreement with the SND data ${ }^{15}$, but in contradiction with DM-2 ${ }^{16}$ measurement.

\section{$3 \pi^{+} \pi^{-} \pi^{+} \pi^{-}, K^{+} K^{-} \pi^{+} \pi^{-}$and} $\boldsymbol{K}^{+} \boldsymbol{K}^{-} \boldsymbol{K}^{+} \boldsymbol{K}^{-}$final states

Event candidates were required to have four good charged tracks and a hard photon assumed to be from ISR. Good PID in BABAR helps to select events with charged kaons. The analysis procedure for $\pi^{+} \pi^{-} \pi^{+} \pi^{-}, K^{+} K^{-} \pi^{+} \pi^{-}$and $K^{+} K^{-} K^{+} K^{-}$final states is described in Ref. ${ }^{13}$. Figure 3 presents the obtained $e^{+} e^{-} \rightarrow \pi^{+} \pi^{-} \pi^{+} \pi^{-}$cross section in comparison with all existing $e^{+} e^{-}$data. The estimated systematic error is about $5 \%$.

The hadronic contribution to $(g-2)_{\mu}$ from this particular channel ${ }^{4}$ evaluated using all available $e^{+} e^{-}$and $\tau$ decay data in $0.56-1.8 \mathrm{GeV}$ range is $\alpha_{\mu}^{\text {hadee }} \cdot 10^{10}=14.21 \pm 0.87_{\text {exp }} \pm 0.23_{\text {rad }}$.

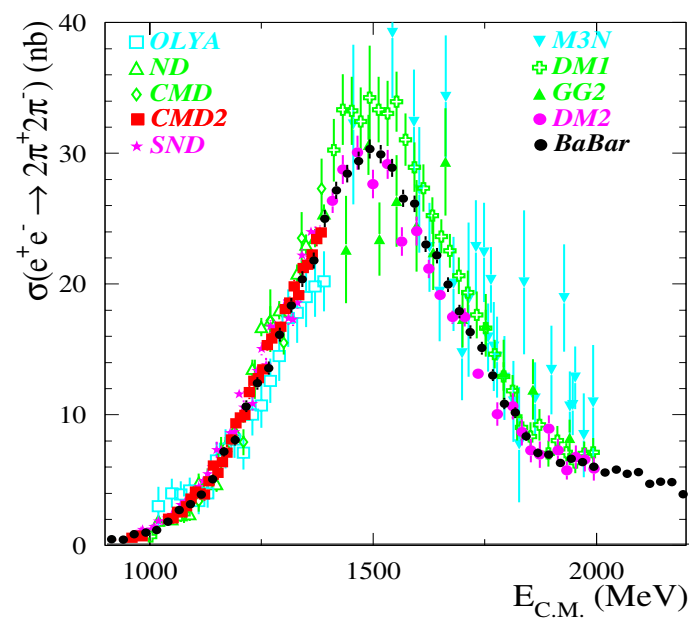

Figure 3. The $e^{+} e^{-} \rightarrow \pi^{+} \pi^{-} \pi^{+} \pi^{-}$cross section obtained from ISR at BABAR in comparison with all $e^{+} e^{-}$data.

$\alpha_{\mu}^{\text {had } \tau} \cdot 10^{10}=12.35 \pm 0.96_{\text {exp }} \pm 0.40_{S U 2}$.

The BABAR data in this energy range give $\alpha_{\mu}^{\text {had }} \cdot 10^{10}=12.95 \pm 0.64_{\text {exp }} \pm 0.13_{\text {rad }}$ which shows the potential of ISR measurements. Figure 4 shows the obtained cross sections for $2 \mathrm{~K} 2 \pi$ and $4 \mathrm{~K}$ final states. The systematic normalization errors are $15 \%$ and $25 \%$ respectively. 

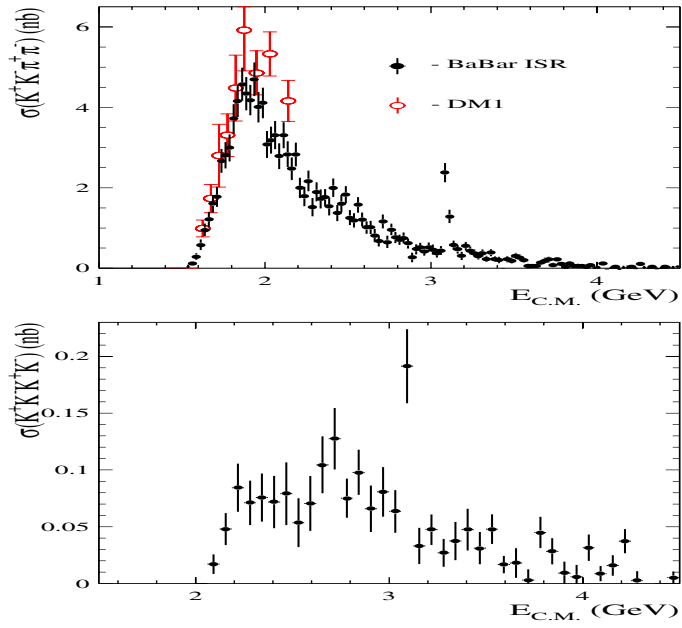

Figure 4. The $e^{+} e^{-} \rightarrow K^{+} K^{-} \pi^{+} \pi^{-}$(top) and $K^{+} K^{-} K^{+} K^{-}$(bottom) cross sections obtained from ISR at BABAR in comparison with $e^{+} e^{-}$data.

\section{The $J / \psi$ decays}

The invariant mass of three pions from $J / \psi$ decay is shown in Fig. 5(top). From about 900 events after background subtraction the following product can be determined:

$\Gamma\left(J / \psi \rightarrow e^{+} e^{-}\right) \mathcal{B}(J / \psi \rightarrow 3 \pi)=$ $(0.122 \pm 0.005 \pm 0.008) \mathrm{keV}$.

The systematic error includes the uncertainties on the detection efficiency, the integrated luminosity, and the radiative correction. Using $\Gamma\left(J / \psi \rightarrow e^{+} e^{-}\right)$measurement from Ref. ${ }^{6}$, the $J / \psi \rightarrow 3 \pi$ branching fraction is calculated to be

$\mathcal{B}(J / \psi \rightarrow 3 \pi)=(2.18 \pm 0.19) \%$,

which is in substantial disagreement $(\sim 3 \sigma)$ with the world average value of $(1.47 \pm$ $0.13) \%$, but agrees with the recent result from the BES ${ }^{17}: \mathcal{B}(J / \psi \rightarrow 3 \pi)=(2.10 \pm 0.12) \%$.

Figure 5 shows the $J / \psi$ and $\psi(2 S)$ signals (containing in $270 \pm 20$ and $620 \pm 25$ observed events respectively) four charged track invariant mass. The later is seen due to the process $\psi(2 S) \rightarrow J / \psi \pi^{+} \pi^{-} \rightarrow \mu \mu \pi^{+} \pi^{-}$and can be easily isolated by requiring a $J / \psi$ mass in one pair of charged particles (shaded histogram). By using detection efficiency from
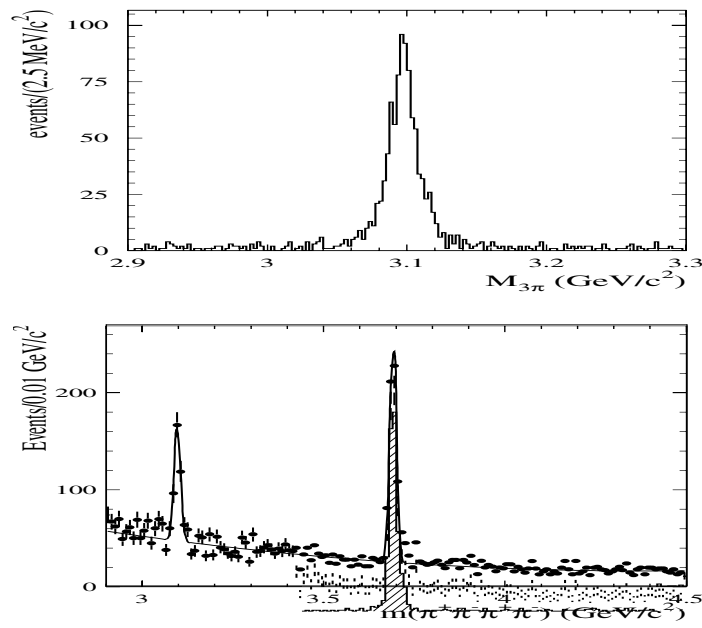

Figure 5. The signals from $J / \psi$ and $\psi(2 S)$ in $3 \pi$ (top) and $4 \pi$ (bottom) final states. The shaded region at the latter corresponds to $\psi(2 S) \rightarrow J / \psi \pi^{+} \pi^{-}$, with $J / \psi \rightarrow \mu^{+} \mu^{-}$.

simulation and the effective ISR luminosity the following products have been obtained:

$\mathcal{B}_{J / \psi \rightarrow 4 \pi} \cdot \Gamma_{J / \psi e e}=$ $(1.95 \pm 0.14 \pm 0.13) \cdot 10^{-2} \mathrm{keV}$, $\mathcal{B}_{\psi(2 S) \rightarrow J / \psi \pi^{+} \pi^{-}} \cdot B_{J / \psi \rightarrow 2 \mu} \cdot \Gamma_{\psi(2 S) e e}=$ $(4.50 \pm 0.18 \pm 0.22) \cdot 10^{-2} \mathrm{keV}$.

Using the world averages value for $\Gamma(J / \psi \rightarrow$ $\left.e^{+} e^{-}\right), \quad \Gamma_{\psi(2 S) \rightarrow e^{+} e^{-}}$and $\mathcal{B}_{J / \psi \rightarrow 2 \mu}$ we derive the values $\mathcal{B}_{J / \psi \rightarrow 4 \pi}=(3.70 \pm$ $0.27 \pm 0.36) \cdot 10^{-3}$ and $\mathcal{B}_{\psi(2 S) \rightarrow J / \psi \pi^{+} \pi^{-}}=$ $0.361 \pm 0.015 \pm 0.037$. Figure 6 shows $J / \psi$ signals in the $K^{+} K^{-} \pi^{+} \pi^{-}$and $K^{+} K^{-} K^{+} K^{-}$ final states. $233 \pm 19$ and $38.5 \pm 6.7$ events have been observed respectively. Using detection efficiency obtained from simulation and effective ISR luminosity the following products have been obtained:

$\mathcal{B}_{J / \psi \rightarrow 2 K 2 \pi} \cdot \Gamma_{J / \psi e e}=$ $(3.29 \pm 0.27 \pm 0.27) \cdot 10^{-2} \mathrm{keV}$, $\mathcal{B}_{J / \psi \rightarrow 4 K} \cdot \Gamma_{J / \psi e e}=$ $(3.6 \pm 0.6 \pm 0.5) \cdot 10^{-3} \mathrm{keV}$.

Using the world average value for $\Gamma(J / \psi \rightarrow$ $\left.e^{+} e^{-}\right)$we derive the relative decay rates $\mathcal{B}_{J / \psi \rightarrow 2 K 2 \pi}=(6.25 \pm 0.50 \pm 0.62) \cdot 10^{-3}$, $\mathcal{B}_{J / \psi \rightarrow 4 K}=(6.9 \pm 1.2 \pm 1.1) \cdot 10^{-4}$. 

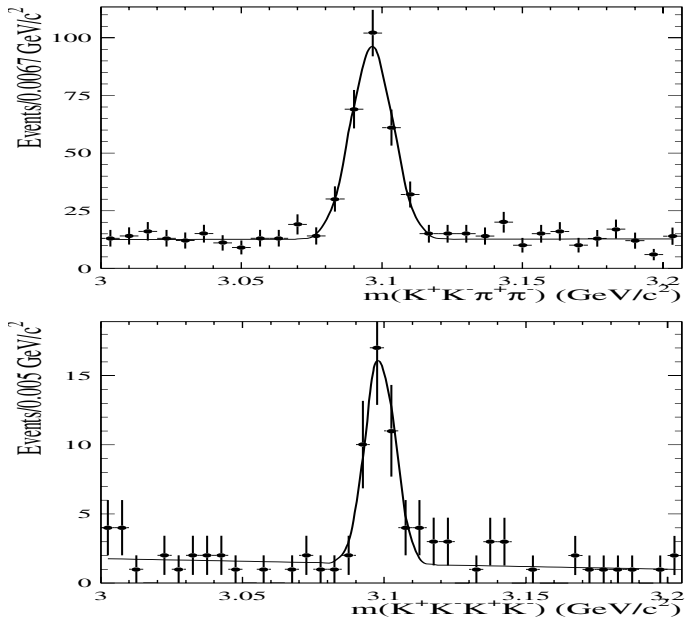

Figure 6. The signals from $J / \psi$ in $2 \mathrm{~K} 2 \pi$ (top) and $4 \mathrm{~K}$ (bottom) invariant masses.

\section{Conclusion}

A number of ISR processes have been studied with $90 \mathrm{fb}^{-1}$ data sample in the $B A B A R$ detector, utilizing the excelent detector efficiency and particle identification capabilities of the detector. The preliminary $e^{+} e^{-} \rightarrow \pi^{+} \pi^{-} \pi^{0}, \pi^{+} \pi^{-} \pi^{+} \pi^{-}$, $K^{+} K^{-} \pi^{+} \pi^{-}, K^{+} K^{-} K^{+} K^{-}$cross sections cover entire mass range from threshold to 4.5 $\mathrm{GeV}$ in the $e^{+} e^{-}$c.m. sistem with systematic normalization errors that are similar to those achived by the direct $e^{+} e^{-}$experiments over a much smaller mass region. Radiative return to the $J / \psi$ resonance allows the measurements of a number of relative branching fractions significantly more precisely than earlier measurements.

\section{References}

1. A.B. Arbuzov et al., JHEP 9812, 009 (1998).

2. S. Binner, J.H. Kuehn, K. Melnikov, Phys. Lett. B459 279 (1999).

3. M. Benayoun et al., Mod. Phys. Lett. A14, 2605 (1999).
4. M.Davier et al., LAL-02-81, Aug 2002. 44pp. e-Print Archive: hep-ph/0208177.

5. E.P.Solodov (for BABAR collaboration), Invited talk at "International Workshop on $e^{+} e^{-}$Physics at Intermediate Energy", SLAC, Stanford, April 30-May 4, 2001, hep-ex/0107027.

6. BABAR Collaboration, B. Aubert et al., Phys. Rev. D 69, 011103 (2004).

7. BABAR Collaboration, B. Aubert et al., hep-ex/0408078. Accepted by PRD.

8. BABAR Collaboration, B. Aubert et al., Nucl. Instr. and Meth. A 479, 1 (2002).

9. H.Czyz and J.H.Kuehn, Eur.Phys.J C18(2000)497-509 (hep-ph/0008262).

10. A.B.Arbuzov et al., JHEP 9710, 001 (1997), hep-ph/9702262.

11. M. Caffo, H. Czyz, E. Remiddi, Nuo. Cim. 110A, 515 (1997); Phys. Lett. B327, 369 (1994).

12. E. Barberio, B. van Eijk and Z. Was. Comput. Phys. Commun. 66, 115 (1991).

13. see talk by R.Stroili in the Proceedings of Hadron2003.

14. Review of Particle Physics, S. Eidelman et al., Phys. Lett. B 592, 1 (2004).

15. SND Collaboration, M.N. Achasov et al., Phys. Rev. D 66, 032001 (2002).

16. DM2 Collaboration, A. Antonelli et al., Z. Phys. C 56, 15 (1992).

17. BES Collaboration, J.Z. Bai et al., Phys. Rev. D 70, 012005 (2004).

18. T. Sjostrand, Comput. Phys. Commun. 82, 74 (1994).

19. S. Jadach and Z. Was, Comput. Phys. Commun. 85, 453 (1995).

20. CMD-2 Collaboration, R.R. Akhmetshin et al., Phys. Lett. B 489, 125 (2000).

21. Crystal Barrel Collaboration, A.V. Anisovich et al., Phys. Lett. B 485, 341 (2000).

22. E852 Collaboration, P. Eugenio et al., Phys. Lett. B 497, 190 (2001).

23. S. Godfrey and N. Isgur, Phys. Rev. D 32, 189 (1985). 